\title{
Synthesis, crystal structure and magnetism of cobalt coordination polymer based on dicyanamide and pyrazine-dioxide derivatives
}

Hao-Ling Sun, Zhe-Ming Wang, Song Gao*

College of Chemistry and Molecular Engineering, State Key Laboratory of Rare Earth Materials

Chemistry and Applications, PKU-HKU Joint Laboratory on Rare Earth Materials and

Bioinorganic Chemistry, Peking University, Beijing 100871, China.

Email: gaosong@pku.edu.cn

Supporting information 


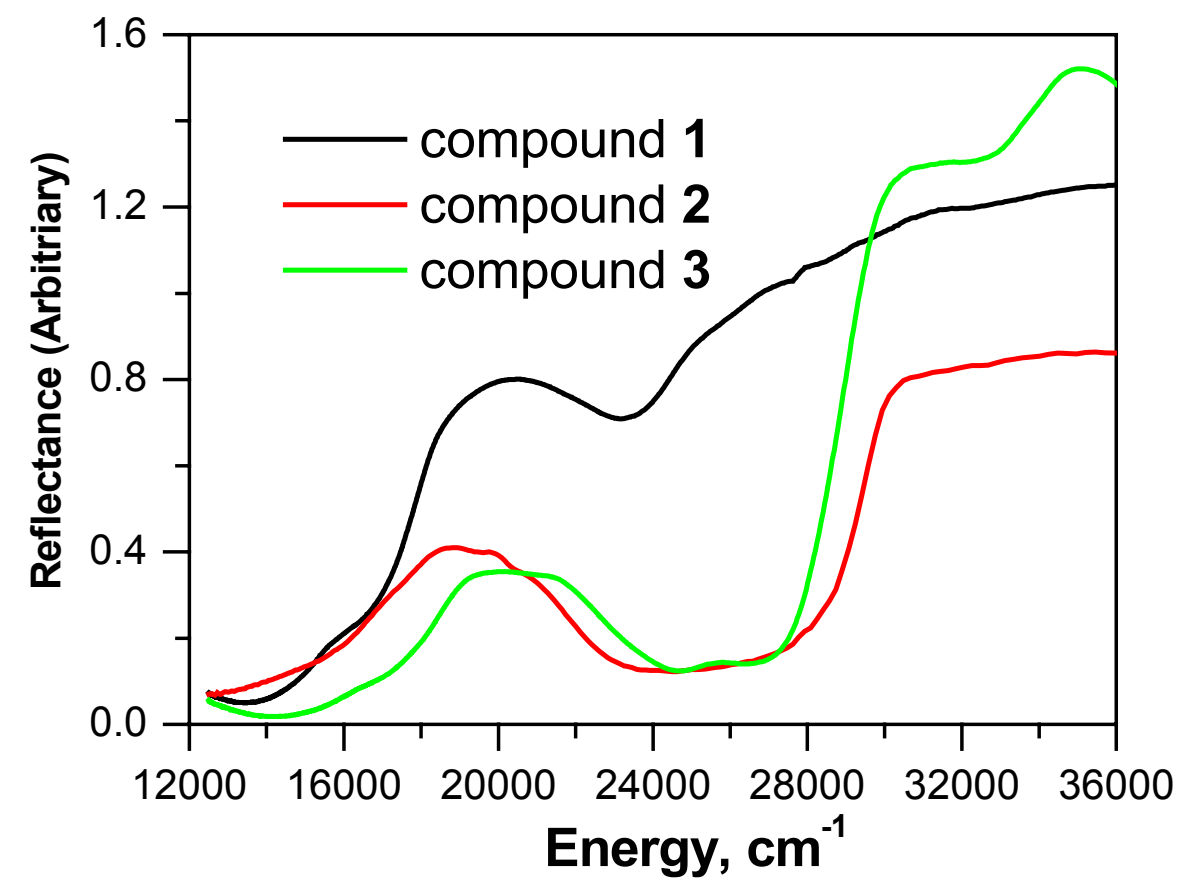

Figure S1. Solid-state UV-visible reflectance spectra of 1, 2, and 3. 


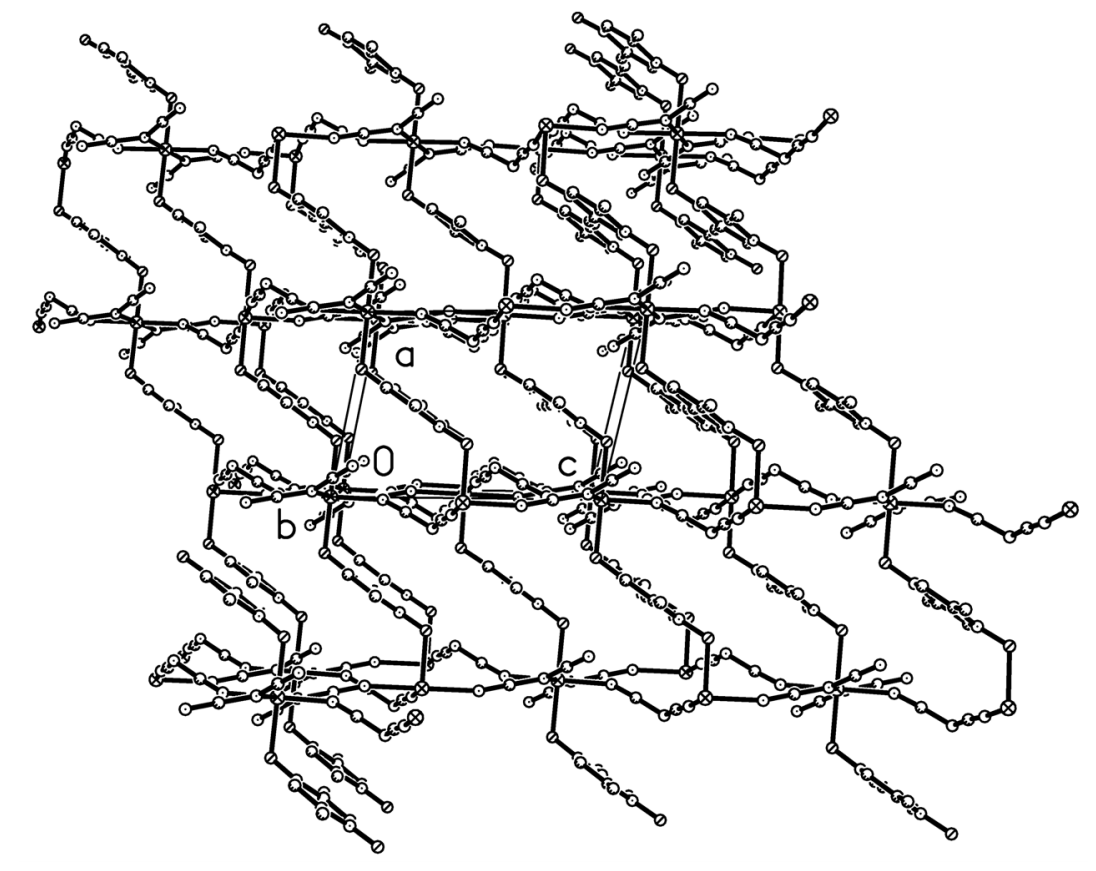

Figure S2. The three-dimensional network constructed by $\mu-2,5$-dmpdo between the parallelogram layers in $\mathbf{1}$. 


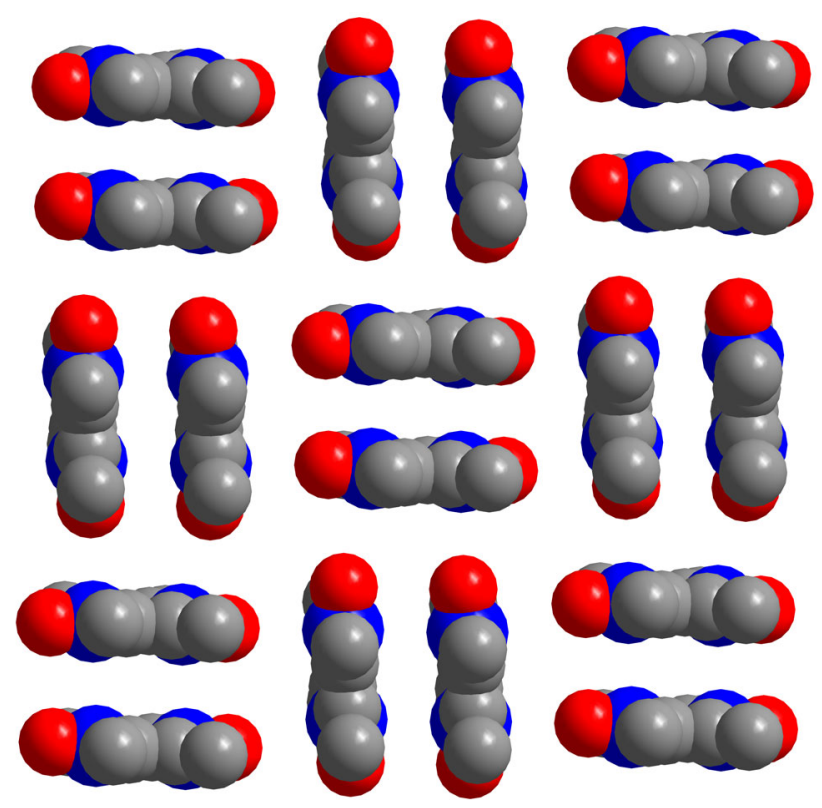

Figure S3. The $\pi \cdots \pi$ stacking pattern of the solvent 2,3,5,6-tmpdo molecular found in 3. 


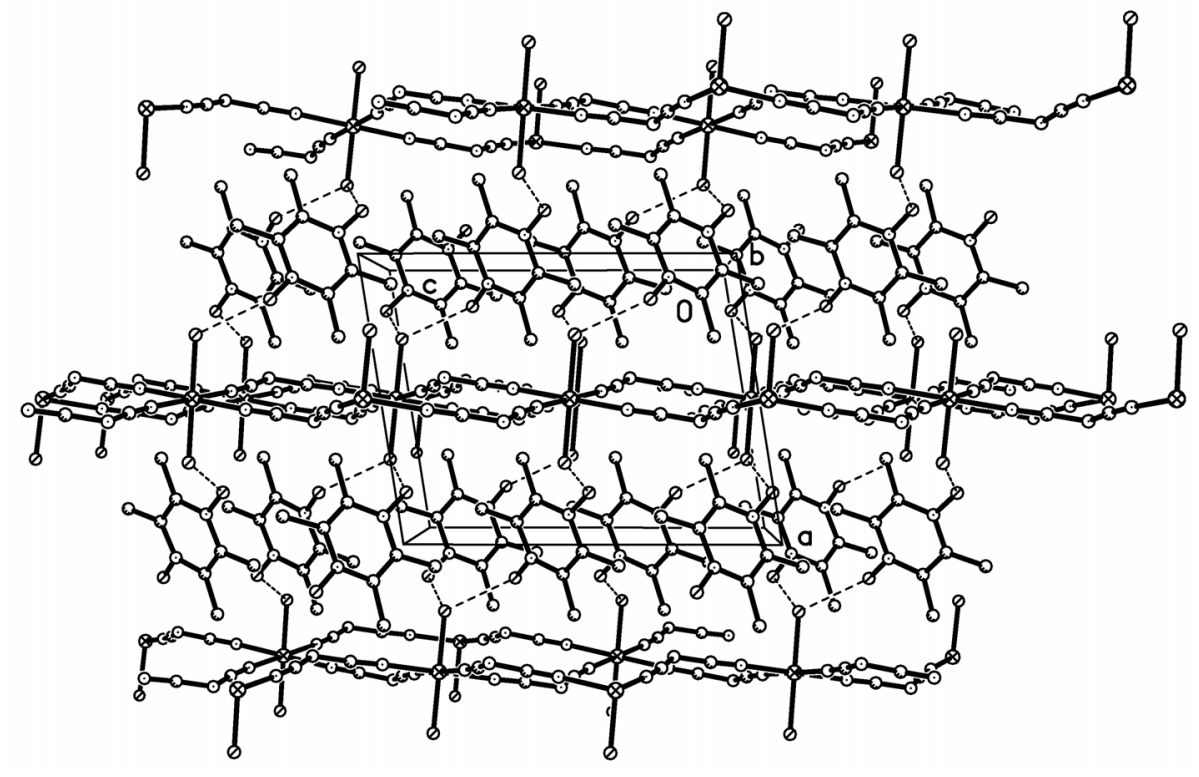

Figure S4. The three-dimensional network constructed by the hydrogen bonding between the 2D $(4,4)$ sheets of 3 


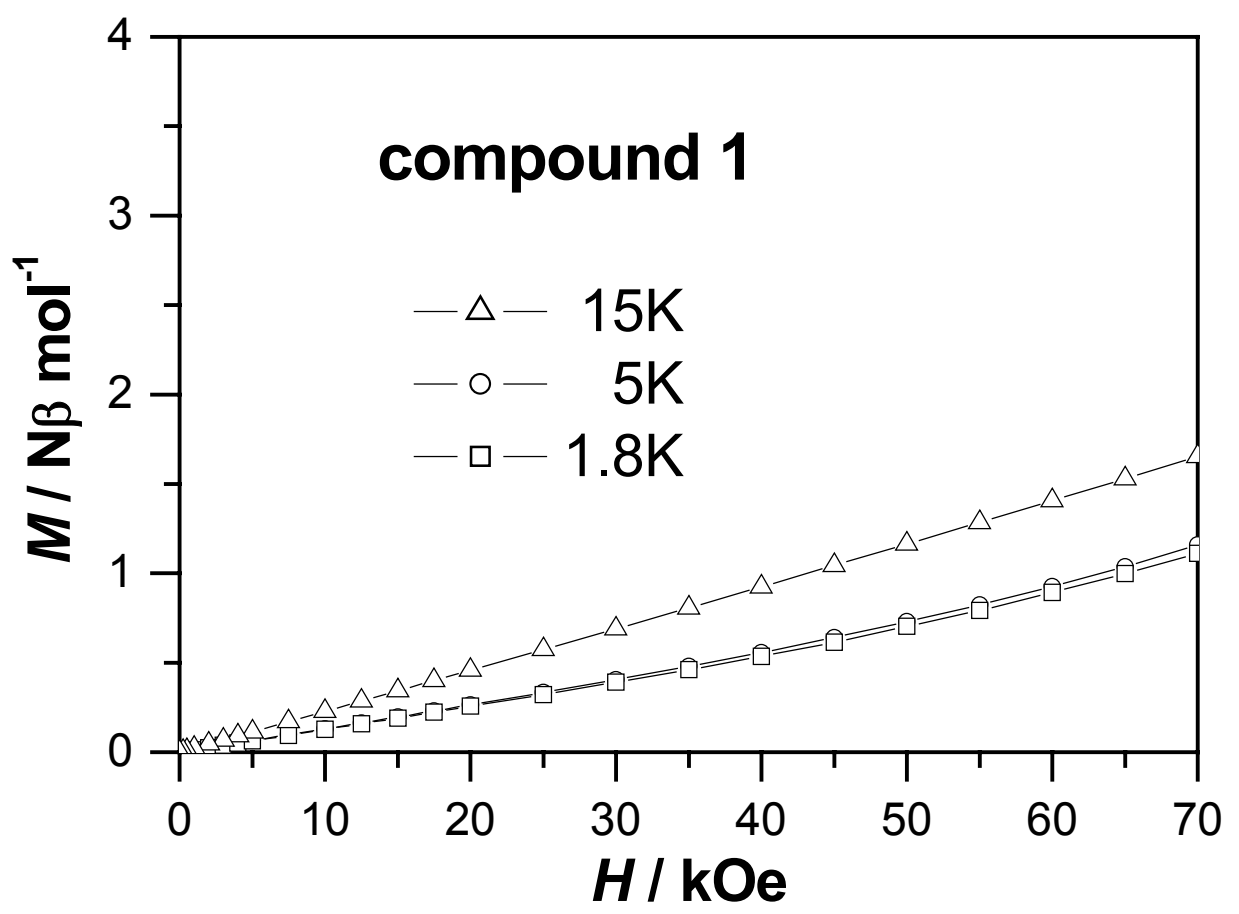

Figure S5. Field dependence of magnetization for 1 measured at $1.8 \mathrm{~K}, 5 \mathrm{~K}$ and $15 \mathrm{~K}$. 


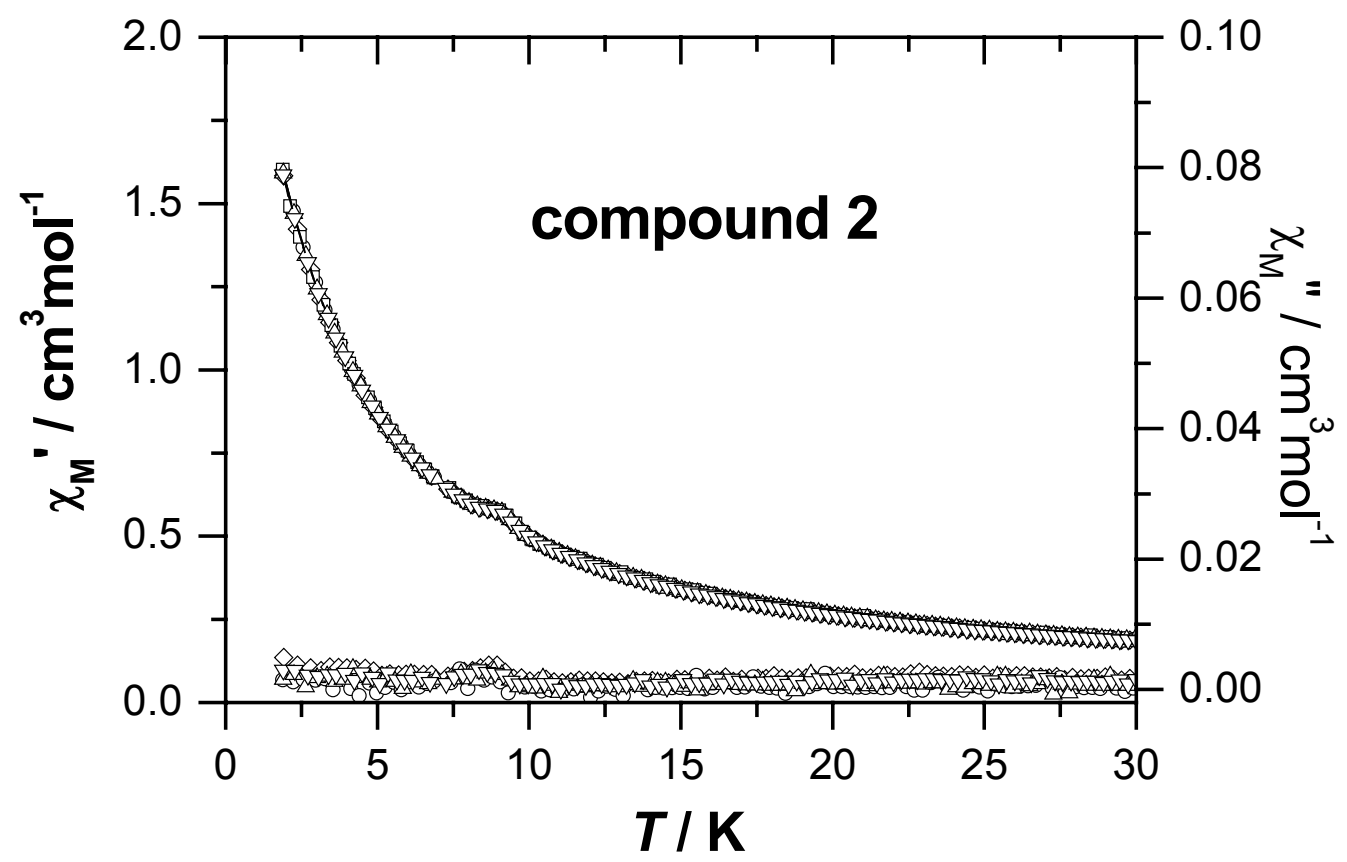

Figure S6. Real ( $\chi$ ') and imaginary ( $\chi$ ”) ac magnetic susceptibilities as a function of temperature taken at 111, 199, 355, 633, and $1111 \mathrm{~Hz}$ for $\mathbf{2}$. 


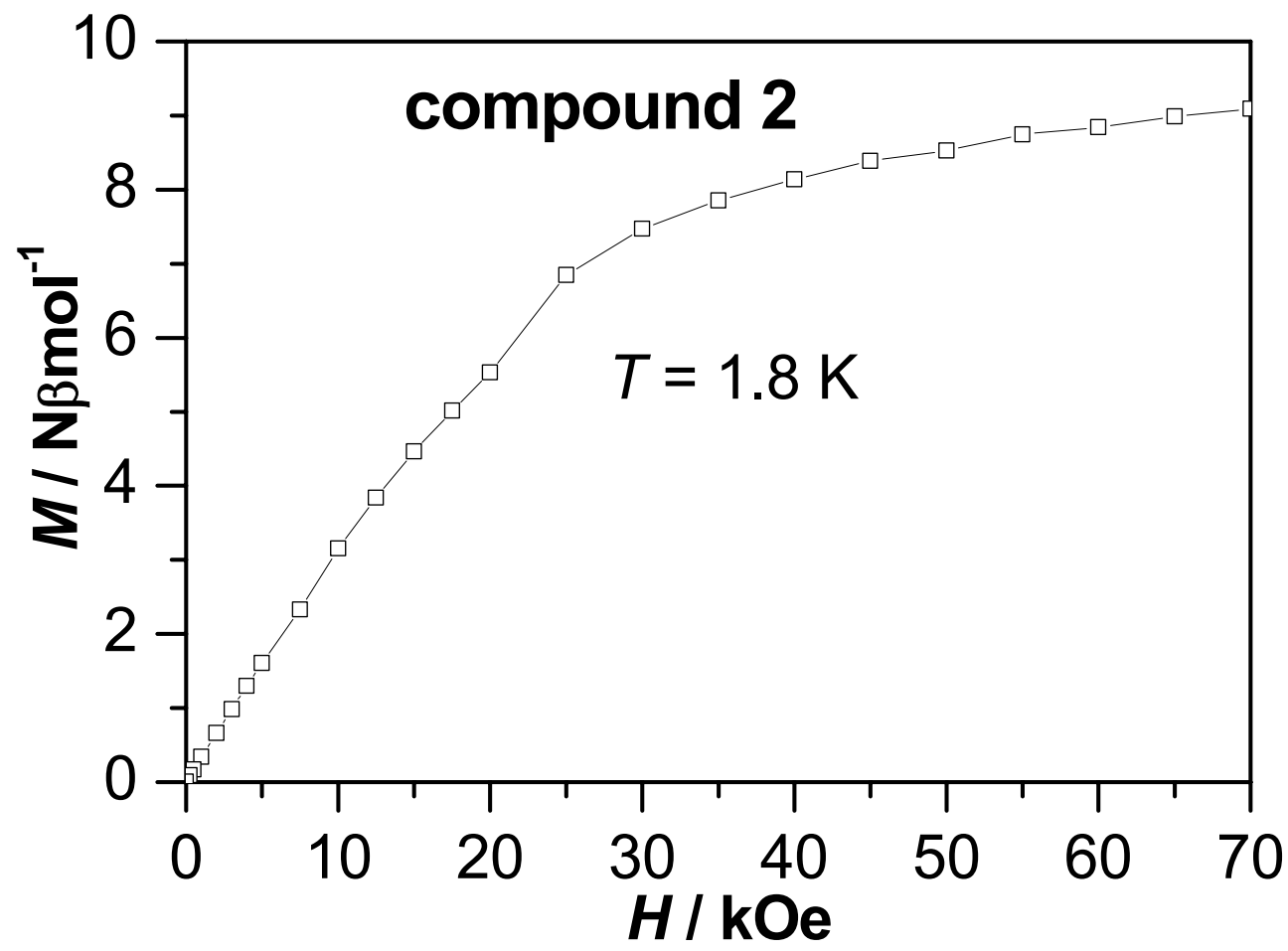

Figure S7. Field dependence of magnetization for 2 measured at $1.8 \mathrm{~K}$. 